\section{Bulk and micropatterned conjugation of extracellular matrix proteins to characterized polyacrylamide substrates for cell mechanotransduction assays}

\author{
Vesna Damljanović, B. Christoffer Lagerholm, and Ken Jacobson \\ University of North Carolina at Chapel Hill, Chapel Hill, NC, USA \\ BioTechniques 39:847-851 (December 2005) \\ doi $10.2144 / 000112026$
}

\begin{abstract}
Increasing numbers of cell mechanotransduction studies are currently utilizing elastic substrates fabricated from polyacrylamide in the form of thin gels. Their versatility depends on the ability to ensure the appropriate gel stiffness and control the uniformity and geometry of extracellular matrix protein coating of the gel. Beginning with a brief quantitative emphasis on the elastic properties of polyacrylamide gels, we present an inexpensive and highly reproducible method for uniform coating with a wide variety of extracellular matrix proteins. We used a reducing agent, hydrazine hydrate, to modify nonreactive amide groups in polyacrylamide to highly reactive hydrazide groups that can form covalent bonds with aldehyde or ketone groups in oxidized proteins. This simple conjugation method overcomes the limitations of previously used photoactivatable cross-linkers: nonuniform coating due to nonuniformity of irradiation and technically challenging procedures for micropatterning. As demonstrated in our study of cell polarity during constrained migration, this conjugation method is especially effective in gel micropatterning by manual microcontact printing of protein patterns as small as 5 um and enables numerous studies of constrained cell attachment and migration that were previously unfeasible due to high cost or difficulty in controlling the protein coating.
\end{abstract}

\section{INTRODUCTION}

During the past decade since the original development of cell traction assays, there has been an increasing interest in mechanical interactions at the cell/substrate interface. Since polyacrylamide gel replaced thin silicone elastomer membrane as a more feasible elastic substrate (1), it has been used not only to measure tractions that cells apply to the substrate during attachment and migration (2), but also to study cell response to varying conditions of extracellular matrix (ECM) (e.g., by varying stiffness) $(3,4)$ and varying electrochemical stimuli (e.g., by the application of an electric field) (5). In all applications, the key steps in gel fabrication are $(i)$ the selection of the appropriate concentrations of monomer and cross-linker to obtain the gel of desired stiffness at given experimental conditions and (ii) uniform and
Inertness of polyacrylamide-a much-cherished property in protein electrophoresis - is the major obstacle in its use as an elastic substrate because it is impossible to covalently couple the gel directly with ECM proteins that are necessary for cell attachment. The currently used conjugation method (1) utilizes a UV-activatable cross-linker, sulfo-SANPAH. The drawbacks of using this expensive reagent are its limited shelf life, poor solubility, rapid decrease of cross-linking activity when solubilized, and dependence on UV lamp power and positioning, resulting in uneven and inconsistent conjugation. In micropatterning, it was satisfactory in combination with patterned elastomer membrane that selectively exposes the UV-activated gel to the protein (8) and in UV-activation through a patterned mask (9), but it failed in the case of the simplest and the most accessible technique (10) - microcontact printing. We have borrowed a surface activation method from oligonucleotide microchips fabrication (11) utilizing hydrazine hydrate, a powerful reducing agent, and modified it in order to obtain a simple method that would be effective for both bulk and micropatterned protein conjugation.

\section{MATERIALS AND METHODS}

\section{Gel Fabrication}

that also lends itself to micropatterning.

It has been known from the early days of gel electrophoresis that the stiffness of polyelectrolyte gel is determined by concentrations of the monomer acrylamide and the crosslinker bis(acrylamide). The effects of gel swelling, ambient temperature, and electrolytic content of aqueous medium have been previously scrutinized (6), but they have not yet received due emphasis in the context of mechanotransduction assays. To provide a broader guideline than that previously reported $(3,7)$ for the selection of appropriate acrylamide/bis concentrations according to required gel stiffness, we have completed extensive measurements of elastic modulus within the range relevant for most cell types.

Potential exfoliation due to swelling and drastic conditions of gel activation necessitate permanent and uniform attachment of the gel to the coverslip. The standard fabrication (1) procedure utilizes smearing of 3aminopropyltrimethoxysilane (Sigma, St. Louis, MO, USA) with a cotton swab over the coverslip surface, but the resulting coating is not uniform enough and does not facilitate mass production. We modified it so as to increase the fabrication output and ensure uniform gel immobilization on the coverslip. After dusting off with compressed air, 12 coverslips (No. 1, $22 \mathrm{~mm}^{2}$; Corning Life Sciences, Acton, MA, USA) were placed on a ceramic rack. The rack was soaked in $0.5 \%$ solution of 3-aminopropyltrimethoxysilane in distilled water for $10 \mathrm{~min}$ on the stir- 
plate. The coverslips were then washed by immersing the rack 3 times in each of the 2 beakers with water and leaving it for $10 \mathrm{~min}$ in a third beaker with water on the stir-plate. The rack was dried at $160^{\circ} \mathrm{C}$ for $1 \mathrm{~h}$. After cooling to room temperature, silanized coverslips were soaked in the rack for 30 min in $0.5 \%$ glutaraldehyde (Electron Microscopy Sciences, Hatfield, PA, USA) in phosphate-buffered saline (PBS) on the stir-plate, washed in water 3 times for $10 \mathrm{~min}$, and dried on the bench. Dried coverslips at either stage of activation can be stored in a desiccator for 2 months.

Gels were prepared using the previously published procedure (1), altering only the concentrations of the initiator and catalyst. The polymerization must occur slowly in order for most of the beads to reach the bottom of the round coverslip (12 or $18 \mathrm{~mm}$ circ.; Fisher Scientific, Fairlawn, NJ, USA) while in the sandwich stage of fabrication, and that was achieved by using the least amounts of initiator ammonium persulfate (Fisher Scientific) and catalyst tetramethylethylenediamine (TEMED; Fisher Scientific) that still provided the desired level of crosslinking in reasonable time. The gel was fully cross-linked with 1:2000 TEMED and 1:250 of 10\% ammonium persulfate after $30 \mathrm{~min}$, resulting with all beads located within $20 \mu \mathrm{m}$ of the top surface. Gels on coverslips were stored individually in $25-\mathrm{mm}$ Petri dishes in PBS at $4^{\circ} \mathrm{C}$.

\section{Protein Conjugation by Gel Hydrazinolysis}

We functionalized polyacrylamide gels by fully immersing a glass rack with 10 gels for at least 2 $\mathrm{h}$ in pure hydrazine hydrate (Sigma). Longer activation times (up to $24 \mathrm{~h}$ ) did not damage the gels, but did not significantly improve the conjugation efficiency. We washed the gels for $1 \mathrm{~h}$ in 5\% glacial acetic acid (Fluka/Sigma) and for $1 \mathrm{~h}$ in distilled water. Activated gels kept in PBS at $4^{\circ} \mathrm{C}$ remained active for at least 2 months.

The gels reported in this paper were coated with the fluorescent Alexa ${ }^{\mathrm{TM}}$ 488 (Molecular Probes, Eugene, OR, USA) conjugate of collagen I (Sigma) or collagen IV (Oregon Green ${ }^{\mathrm{TM}} 488$ conjugate; Molecular Probes), but we tested this method successfully with fluorescent conjugates of other common ECM proteins, such as fibronectin and laminin, and also bovine serum albumin (BSA), a standard negative control in cell attachment studies (data not shown).

We solubilized the collagen in 0.1 $\mathrm{M}$ acetic acid, and fibronectin, laminin, and albumin in PBS, each to a concentration of $1 \mathrm{mg} / \mathrm{mL}$. In order to enable oxidation, we diluted the collagen solution in $50 \mathrm{mM}$ sodium acetate buffer (EM Science, Gibbstown, NJ, USA) to $0.1 \mathrm{mg} / \mathrm{mL}$ with $\mathrm{pH} 4.5$. The oxidation was achieved by adding 3.6 $\mathrm{mg} / \mathrm{mL}$ sodium periodate (Sigma) crystals and incubating at room temperature for $30 \mathrm{~min}$. When larger volumes were to be oxidized and kept for later use, the sodium periodate was added at lower concentrations (up to $3.2 \mathrm{mg}$ /
$\mathrm{mL})$. The same procedure was used for all other tested proteins.

For bulk conjugation, a small volume (e.g., $0.5 \mathrm{~mL}$ for a 18 -mm diameter gel) of oxidized collagen was pooled onto the activated gel surface, incubated at room temperature for $1 \mathrm{~h}$, and washed in PBS. For patterned conjugation, 50 $\mu \mathrm{L}$ of oxidized collagen were pooled on the patterned side of a $5 \times 5 \mathrm{~mm}$ poly(dimethylsiloxane) (PDMS) stamp (donated by Superfine Laboratory, Department of Physics, University of North Carolina at Chapel Hill, Chapel Hill, NC, USA) and incubated for 45 min. PDMS has a low surface energy and strong affinity for hydrophobic parts of molecules, so incubation leaves a monolayer of protein adhering weakly to the stamp. After blowing the excess solution off the stamp with a nitrogen gun, the stamp was manually placed in contact with the gel, which was previously dried for $15 \mathrm{~min}$ and lifted off
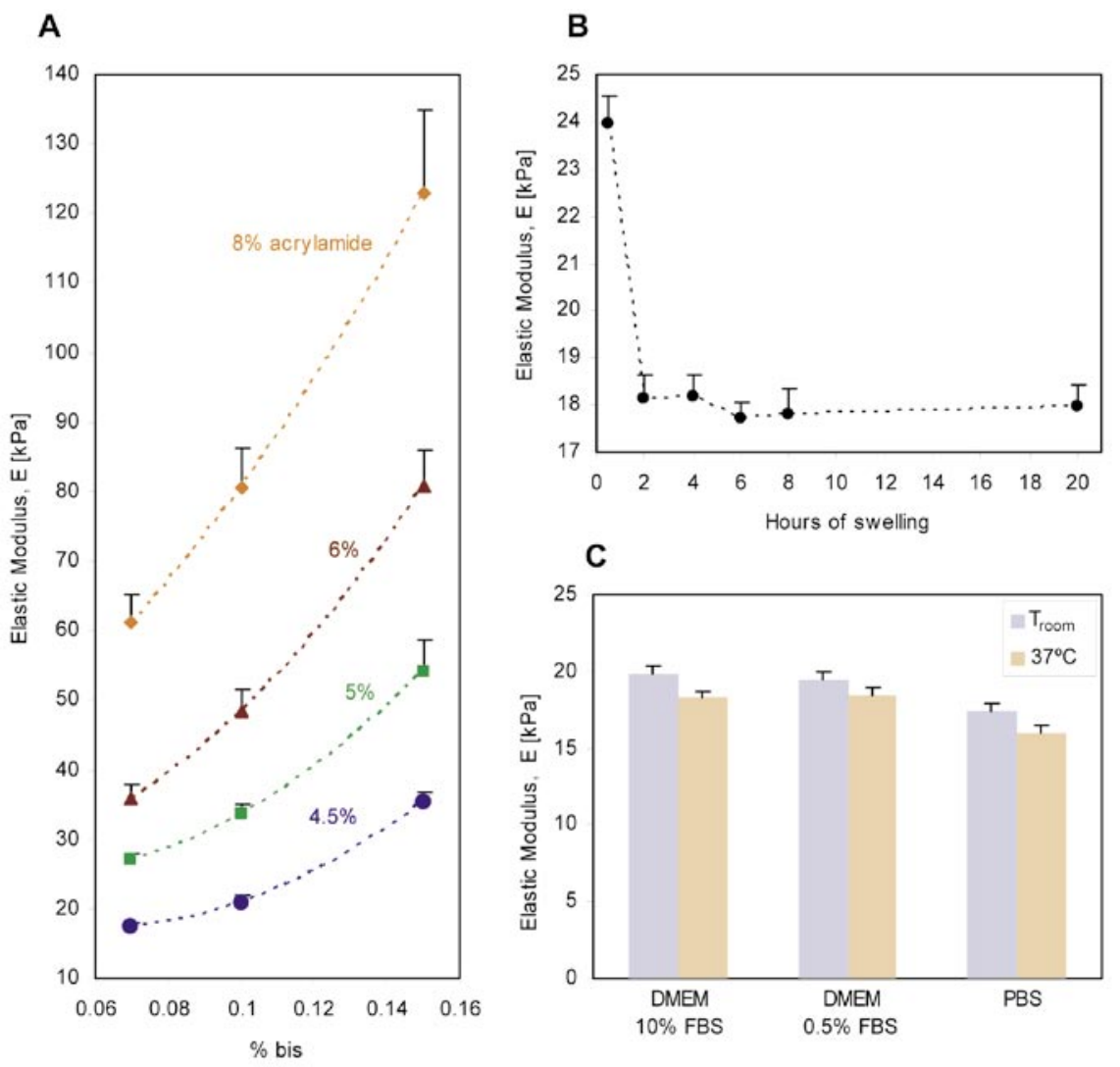

Figure 1. Effects of environment and cross-linking on modulus. (A) Stiffness versus bis concentration (dashed lines: quadratic fit). Gels with $4.5 \%$ acrylamide, $0.07 \%$ bis: (B) decrease in stiffness during swelling in PBS at room temperature; (C) stiffness versus media type and temperature. All error bars indicate the SEM. $T_{\text {room }}$, room temperature; PBS, phosphate-buffered saline; DMEM, Dulbecco's modified Eagle's medium; FBS, fetal bovine serum. 
after $30 \mathrm{~s}$. The gel was then washed as in the protocol for bulk conjugation.

\section{Measurement of Gel Elastic Modulus}

We calculated the modulus from the automatized measurement of depth of indentation by a $0.5-\mathrm{mm}$ diameter carbide pen ball (Hoover Precision Products, East Granby, CT, USA) using the Hertzian pressure method (1) that, if carefully employed, is an accurate and simple alternative for the highly accurate but often inaccessible atomic force microscopy (AFM) method, which also suffers from the same limitations (sensitive to surface impurities) as its simpler cousin. We rejected the other two available methods-probing with a microneedle and the macroscopic uniaxial tension test-because the first is often damaging to the gel and requires extensive computations, and the second, although analytically simpler, is affected by difficulties in weight attachment and in maintaining the temperature and hydration at the same levels as during the actual assays.

Hertzian pressure theory draws the relationship between the stiffness of a compliant material and the depth of indentation due to the weight of the ball of a much stiffer material, with adjustment for buoyancy. If a steel or carbide ball of radius $r$ and density $\rho_{b}$, dropped through the fluid medium of density $\rho_{f}$, falls on the top of the polyacrylamide gel of Poisson ratio $v$ (assumed to be 0.3 ), creating the indentation of the depth $d$, the elastic modulus (or Young's modulus, or simply stiffness) is, in the first approximation, $\mathrm{E}=\pi\left(1-v^{2}\right) d^{-3 / 2} r^{5 / 2}$ $g\left(\rho_{b}-\rho_{f}\right)$, where $g$ is the gravitational acceleration, $9.81 \mathrm{~m} / \mathrm{s}^{2}$. If there are no surface defects or impurities near the indentation, the method provides a reliable measure of gel stiffness.

The key to the accuracy of this method lies in the measurement of the vertical position of the same point on the gel surface with and without the steel ball on it. The detailed procedure that gave the best results is explained in the online Supplementary Material available at www.BioTechniques.com (Figure S1). We were generally able to conduct our indentation depth measure- ments with at least $\pm 0.3 \mu \mathrm{m}$ accuracy, which gives very accurate modulus measurements for more compliant gels (deeper indentation, large $d$ ) and less accurate for stiffer gels (shallow indentation, small $d$ ). The estimated relative error for the modulus measurements shown in this paper was in the range of $4 \%$ for the most compliant gels to $14 \%$ for the stiffest gels. This estimate is a pointer for what to expect if using a similar measurement setup.

\section{Cell Culture}

Mouse embryo $\mathrm{C} 3 \mathrm{H}$ fibroblasts were cultured at $37^{\circ} \mathrm{C}$ in Dulbecco's modified Eagle's medium with F12 nutrient (DMEM-F12; Invitrogen, Carlsbad, CA, USA) supplemented with $10 \%$ fetal bovine serum (FBS; Atlanta Biological, Lawrenceville, GA, USA) and 1\% antibiotic-antimycotic
(Invitrogen). Cells were plated on the gel at a 1:20 dilution of a 70\% confluent 60-mm Petri dish and incubated for 2 $\mathrm{h}$ prior to the experiment. For toxicity analysis, the cells were kept on gels in the incubator without changing the medium for up to 7 days (when only $20 \%$ of the original $3 \mathrm{~mL}$ of media still remained).

\section{Traction Assays}

The maps of gel deformation due to cell tractions for each time point were obtained from images of fluorescent beads $(0.2 \mu \mathrm{m})$ on the gel surface using the correlation-based optical flow method (12). The traction maps were calculated according to the established method (2) using the LIBTRC software (M. Dembo, Boston University, Boston, MA, USA).
A

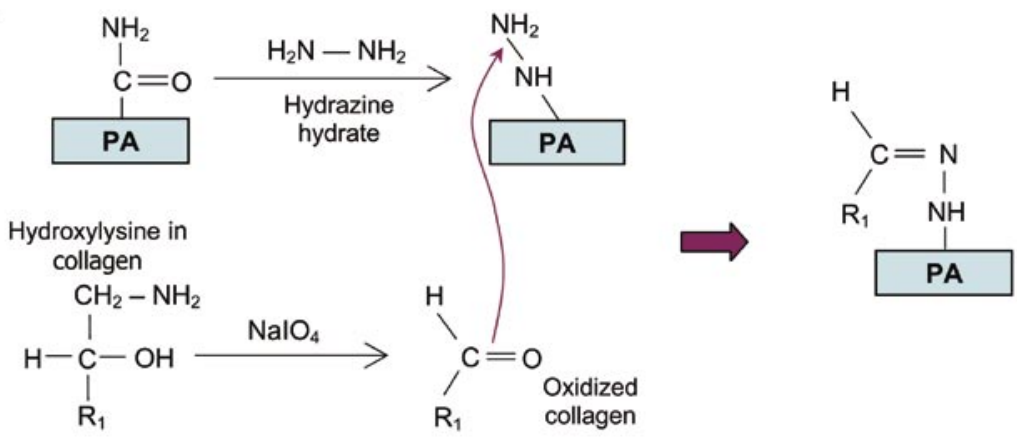

Figure 2. Gel conjugation with collagen. (A) Diagram of polyacrylamide hydrazinolysis and conjugation of oxidized collagen. Microprinted collagen IV patterns: (B) $18 \mu \mathrm{m}$ stripes, $4 \mu \mathrm{m}$ spacing; (C) 16 $\mu \mathrm{m}$ stripes, $4 \mu \mathrm{m}$ spacing; (D) $130 \times 138 \mu \mathrm{m}$ rectangles. (E) $\mathrm{C} 3 \mathrm{H}$ fibroblasts on $15 \mu \mathrm{m}$ stripes of collagen I. (F) Healthy $\mathrm{C} 3 \mathrm{H}$ fibroblasts 3 days after plating on the gel partially conjugated with collagen I (collagen is on bottom half of image, top half is inert). (G) Traction map of $\mathrm{C} 3 \mathrm{H}$ fibroblast moving along a $25-\mu \mathrm{m}$ wide stripe (outline traced in black) of collagen I; full and dashed arrows indicate original and reversed direction, respectively. PA, polyacrylamide. 


\section{RESULTS AND DISCUSSION}

\section{Cross-Linking and Environment Affect Gel Stiffness}

Increasing the concentration of either acrylamide or bis results in greater elastic modulus (i.e., stiffness) (Figure 1A). The choice of the appropriate acrylamide/bis concentrations must be informed by two additional facts: $(i)$ that the polyacrylamide gel swells during hydration in cell culture medium, becoming more compliant at equilibrium state if the gel has not fully cross-linked before flooding with buffer as it was the case here (Figure 1B); and (ii) that the modulus decreases with an increase in temperature and depends on the ionic strength of the medium (Figure 1C). The elastic modulus is slightly lower in PBS than in DMEM, which is to be expected (6), considering the slightly higher ionic content of PBS (0.193) than that of DMEM (0.169). The concentration of serum in the media does not seem to affect the modulus, which is not surprising, considering that serum does not contain additional salts. Various gel treatments associated with protein conjugation also have no discernable effect on gel stiffness (data not shown).

Note that the strict following of the fabrication protocol, even by the same individual, does not guarantee consistent elastic moduli. The amount of mixture lost during degassing is unpredictable. The mixing of minute amounts of catalyst and initiator in orders of magnitude greater volume of mixture is uneven and results in nonuniform cross-linking across the volume, from which only a small amount is then drawn to cast the gel. As the remaining shelf life of catalyst and initiator decreases, higher concentrations of both are needed to achieve the same gel stiffness. These unavoidable issues affect gel stiffness enough to account for the large inconsistencies in modulus measurements published to date. Until these gels become available commercially and are consequently standardized, it is necessary to measure the elastic modulus of each gel or at least one gel per batch, always under actual experimental conditions.

\section{Hydrazinolysis Increases Protein Conjugation Efficiency}

Treatment with hydrazine hydrate modifies nonreactive amide groups in the polyacrylamide gels to hydrazide groups, which in turn are highly reactive toward proteins that contain either aldehyde or ketone groups (Figure 2A, top) (13). Because glycosylated proteins, such as collagen and other ECM proteins, are easily modified to contain aldehyde groups by oxidation with sodium $m$-periodate (14), this method offers a very efficient and simple conjugation method to polyacrylamide gels (Figure 2A, bottom). In addition to glycosylated proteins, this method will also work with proteins containing a primary amine and a secondary hydroxyl on adjacent carbon atoms as is the case with hydroxylysine, which is prevalent in collagen, and $\mathrm{N}$-terminal serine and threonine residues (15).

The uniformity of bulk coating was confirmed by uniformly bright images (data not shown) of gels conjugated with Oregon Green-labeled collagen, which was also used to visualize the stamped patterns. The success of this method is best seen by considering the excellent one-to-one transfer of complex patterns, including all the stamp imperfections (rims in Figure 2D) and the striking sharpness of edges and features as small as $10 \mu \mathrm{m}$. Remarkably, the binding is instantaneous, as indicated by the immaculate transfer of pattern (Figure 2, B-D) despite inevitable stamp slipping due to manual stamping. Although pure hydrazine hydrate is toxic, neutralization with acetic acid leaves no trace of toxicity, as indicated by fibroblasts with normal phenotype and viability 3 days after being plated on the gel with micropatterned collagen (Figure 2, E and $F$ ). The lack of nonspecific binding in inert areas not printed with collagen is also indicated.

\section{Current and Future Applications in Mechanotransduction Studies}

One application example of polyacrylamide micropatterning using this method is our current study of cell polarity during migration. Traction maps of constrained cells have been previously reported, but only during stationary attachment (9) and not during migration. Using the previously developed traction assay (2), we measured tractions that fibroblast applies on the substrate while moving along a stripe of protein $25 \mu \mathrm{m}$ wide that confines the cell to one-dimensional (1-D)-like migration (Figure $2 \mathrm{G})$. We captured the moment when the cell reached the end of stripe and repolarized in order to start moving in the opposite direction, as indicated by the redistribution of high tractions from the front to the back. This and numerous other studies with micropatterned elastic substrates are rendered more affordable by simple conjugation of oxidized protein to hydrazinolyzed polyacrylamide gels.

In summary, this method of protein conjugation has many advantages compared to using sulfo-SANPAH. Simultaneous bulk hydrazinolysis of many gels ensures greater uniformity and repeatability of protein conjugation, and the bulk conjugation time is shorter ( $1 \mathrm{~h}$ versus overnight on a shaker). Gels activated with hydrazine hydrate remain active for months, as opposed to a few minutes for sulfo-SANPAH. Furthermore, oxidized protein remains stable at $4^{\circ} \mathrm{C}$ for as long as an untreated protein. Hydrazinolyzed gels can be mass produced, stored at any stage of fabrication, and easily shipped. Finally, this simple and inexpensive method is useful both in microprinting and in covering large surfaces (e.g., for experiments requiring cell lysate). When used for microprinting combined with varied gel stiffness, it can become a powerful tool for the study of cell mechanotransduction.

\section{ACKNOWLEDGMENTS}

This work was supported by the National Institutes of Health (NIH) Cell Migration Consortium. PDMS stamps were kindly provided by Lloyd Carroll. Many thanks for illuminating discussions go to Yu-Li Wang, Micah Dembo, and Vytas Bankaitis. 


\section{COMPETING INTERESTS STATEMENT}

The authors declare no competing interests.

\section{REFERENCES}

1. Beningo, K.A., C.-M. Lo, and Y.-L. Wang. 2002. Flexible polyacrylamide substrata for the analysis of mechanical interactions at cell-substratum adhesions. Methods Cell Biol. 69:325-339.

2. Dembo, M. and Y.-L. Wang. 1999. Stresses at the cell-to-substrate interface during locomotion of fibroblasts. Biophys. J. 76:23072316.

3.Pelham, R.J. and Y.-L. Wang. 1997. Cell locomotion and focal adhesions are regulated by substrate flexibility. Proc. Natl. Acad. Sci. USA 94:13661-13665.

4. Gray, D.S., J. Tien, and C.S. Chen. 2003. Repositioning of cells by mechanotaxis on surfaces with micropatterned Young's modulus. J. Biomed. Mater. Res. A. 66:605-614.

5. Curtze, S., M. Dembo, M. Miron, and D.B. Jones. 2004. Dynamic changes in traction forces with DC electric field in osteoblast-like cells. J. Cell Sci. 117:2721-2729.

6. Schröder, U.P. and W. Oppermann. 1996. Properties of polyelectrolyte gels, p. 1938. In J.P. Cohen Addad (Ed.), The Physical Properties of Polymeric Gels. John Wiley \& Sons, New York.

7. Engler, A., L. Bacakova, C. Newman, A. Hategan, M. Griffin, and D. Discher. 2004. Substrate compliance versus ligand density in cell on gel responses. Biophys. J. 86:617-628.

8. Parker, K.K., A.L. Brock, C. Brangwynne, R.J. Mannix, N. Wang, E. Ostuni, N.A. Geisse, J.C. Adams, et al. 2002. Directional control of lamellipodia extension by constraining cell shape and orienting cell tractional forces. FASEB J. 16:1195-1204.

9. Wang, N., E. Ostuni, G.M. Whitesides, and D.E. Ingber. 2002. Micropatterning tractional forces in living cells. Cell Motil. Cytoskeleton 52:97-106.

10.Whitesides, G.M., E. Ostuni, S. Takayama, X. Jiang, and D.E. Ingber. 2001. Soft lithography in biology and biochemistry. Annu. Rev. Biomed. Eng. 3:335-373.

11.Timofeev, E., S.V. Kochetkova, A.D. Mirzabekov, and V.L. Florentiev. 1996. Regioselective immobilization of short oligonucleotides to acrylic copolymer gels. Nucleic Acids Res. 24:3142-3148.

12.Marganski, W.A., M. Dembo, and Y.-L. Wang. 2003. Measurements of cell-generated deformations on flexible substrata using correlation-based optical flow. Methods Enzymol. 361:197-211.

13.Hermanson, G.T. 1996. Part I, section 4.5. Introduction to hydrazide functional groups, p. 120-125. In Bioconjugate Techniques. Academic Press, San Diego, CA.

14.Fischer, B.E., K.B. Thomas, and F. Dorner. 1998. Collagen covalently immobilized onto plastic surfaces simplifies measurement of von Willebrand factor-collagen binding activity. Ann. Hematol. 76:159-166.

15.Geoghegan, K.F. and J.G. Stroh. 1992. Sitedirected conjugation of nonpeptide groups to peptides and proteins via periodate oxidation of a 2-amino alcohol. Application to modification at $\mathrm{N}$-terminal serine. Bioconjug. Chem. 3:138-146.

Received 4 July 2005; accepted 29 July 2005.

Address correspondence to Vesna Damljanović, Biomedical Engineering Department, Boston University, 44 Cummington Street, Boston, MA 02215, USA.e-mail:damljano@bu.edu

To purchase reprints of this article, contact Reprints@BioTechniques.com 